\title{
Sound propagation through a rarefied gas in rectangular channels
}

\author{
Lei $\mathrm{Wu}^{1}$ \\ ${ }^{1}$ James Weir Fluids Laboratory, Department of Mechanical and Aerospace Engineering, \\ University of Strathclyde, Glasgow G1 1XJ, UK
}

\begin{abstract}
A sound propagation through a rarefied gas inside a two-dimensional cavity is investigated on the basis of the linearized Boltzmann equation, where one of the cavity wall oscillates harmonically in the normal direction to its own surface and is considered as a sound source. An analytical solution at high oscillation frequencies is obtained, and detailed numerical results for a wide range of gas rarefaction are presented. The influence of both the aspect ratio of the cavity and the oscillation frequency on the average gas pressure exerted on the oscillating plate is studied. It is found that, at large values of the aspect ratio, the average pressure oscillates when the sound frequency varies, due to the sound resonance and anti-resonance along the oscillation direction of the plate. However, at small values of the aspect ratio, the average pressure is a monotonically decreasing function of the sound frequency, which cannot be observed in the corresponding one-dimensional counterpart. This is explained by the sound interference in the direction parallel to the oscillating plate. The influence of both the cavity aspect ratio and oscillation frequency on the sound speed is also investigated: again it is found that different aspect ratio leads to the different behavior of the sound speed as a function of the oscillation frequency.
\end{abstract}

\section{INTRODUCTION}

The study of rarefied gas flows is important for a broad range of industrial applications, and has attracted significant attention due to the rapid development of microelectromechanical systems (MEMS) [1]. As the systems approach the micro/nano scale, the Navier-Stokes equations, based on the continuum-fluid hypothesis, become invalid. The Knudsen number $K n$, defined as the ratio of the mean free path of gas molecules to the characteristic system length, is usually adopted to quantify the deviation from the continuum behavior [2]. The gas flow is in the continuum regime when the Knudsen number is less than 0.001. When $0.001 \lesssim K n \lesssim 0.1$, the gas flow is in the slip regime, where the NS equations with the velocity slip and temperature jump boundary conditions may still be valid. When $0.1 \lesssim K n \lesssim 10$ and $K n \gtrsim 10$, the gas flows are in the transition and freemolecular regimes, respectively, where counter-intuitive phenomena arise due to the rarefaction effects [3-5], including the nonlinear stress/strain-rate behavior, the "Knudsen paradox" where the dimensionless mass flow rate in Poiseuille flow could increase when the gas pressure decreases [6,7], and the thermal transpiration where gas molecules move from the cold region to hot [8-10]. At the standard pressure and temperature, air has a molecular mean free path of about $68 \mathrm{~nm}$, which is comparable to the length of a MEMS device, and the Boltzmann equation must be used to capture the rarefaction effects.

The problem becomes even complicated in oscillatory gas flows, where the deviation from the continuum behavior is not only determined by the Knudsen number, but also by the ratio of the characteristic oscillation frequency to the mean molecular collision frequency. Even at small Knudsen numbers, the oscillatory gas flow can not be properly described by Navier-Stokes equations when the oscillation frequency is comparable to or even higher than the mean molecular collision frequency $[11,12]$.
Oscillatory gas flows are common in MEMS devices, and the investigation of the damping force exerted by the gas to the oscillatory parts of a MEMS device is important in a number of applications such as inertial sensing and acoustic transduction. In the past decade, oscillatory gas flows have been extensively studied [1120], most of which, however, are for flows between two parallel plates. While the viscous damping is dominate at low oscillation frequencies, at relatively high oscillation frequencies, inertial force leads to the interference of sound waves along the oscillating direction of the plate, so that the magnitude of the damping force on the oscillating plate oscillates when the oscillation frequency varies [18]. This interference introduces new phenomena in oscillatory rarefied gas flows; for instance, in the twodimensional cavity flow, due to the anti-resonance, the damping force on the oscillating lid can even be smaller than that in the one-dimensional counterpart, when the cavity aspect ratio is properly chosen [21].

In this paper, we study the sound propagation inside a two-dimensional cavity, where the sound is generated by the oscillation of one of the cavity wall. We investigate the influence of both the cavity aspect ratio and the oscillation frequency on the damping force exerted on the oscillating plate and phase speed of the sound. A novel resonance, perpendicular to the oscillating direction of the plate, is observed and analyzed, through the analytical calculation and numerical simulation of the linearized Boltzmann equation (LBE). The threshold of the cavity aspect ratio that leads to the new resonance mechanism is also obtained.

\section{STATE OF THE PROBLEM}

We consider a rarefied flow of a monatomic gas inside a cuboid, driven by a plate at $x=0$ that oscillates harmonically in the $x$ direction with the frequency $\omega$, while 


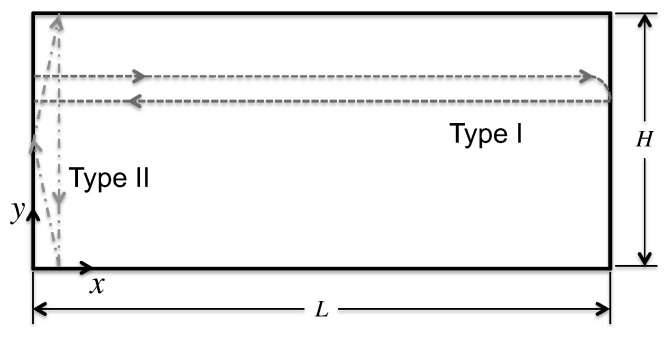

FIG. 1. Cavity geometry and two types of interference. The left plate oscillating harmonically in the $x$ direction is considered as the sound source. Tpye I and II interference occur in the direction parallel and perpendicular to the motion of the oscillating plate, respectively.

the other walls are fixed. All the walls of the cuboid are held at the same constant temperature $T_{0}$. The length of the cuboid in the $z$ direction is much larger than the width $L$ and height $H$ in the $x$ and $y$ directions, respectively, so that the flow is effectively two-dimensional, see Fig. 1. The velocity of the oscillating plate depends on time $t$ through the formula

$$
U_{w}=\Re\left[U_{0} \exp (i \omega t)\right],
$$

where $i$ is the imaginary unit and $\Re$ denotes the real part of a complex expression. The velocity amplitude $U_{0}$ is assumed to be very small when compared to the most probable speed $v_{m}$ of the gas molecules, i.e.,

$$
U_{0} \ll v_{m}, \quad v_{m}=\sqrt{\frac{2 k_{B} T_{0}}{m}},
$$

where $k_{B}$ is the Boltzmann constant and $m$ is the molecular mass of the gas.

The induced oscillatory rarefied gas flows are characterized by the cavity aspect ratio

$$
A=\frac{H}{L}
$$

the Strouhal number

$$
S=\frac{\omega L}{v_{m}},
$$

and the Knudsen number

$$
K n=\frac{\lambda}{L}=\frac{\mu}{n_{0} L} \sqrt{\frac{\pi}{2 m k_{B} T_{0}}},
$$

where $\lambda$ is the molecular mean free path, $n_{0}$ is the molecular number density in equilibrium, and $\mu$ is the shear viscosity of the gas at the reference temperature $T_{0}$.

We use the Boltzmann equation to describe the rarefied gas dynamics, which can be linearized due to the fact that the deviation from the global equilibrium state is small: the velocity distribution function, normalized by $n_{0} / v_{m}^{3}$, can be expressed as

$$
f(\mathbf{v}, x, y, t)=f_{e q}(\mathbf{v})+h^{\prime}(\mathbf{v}, x, y, t),
$$

where $\mathbf{v}=\left(v_{x}, v_{y}, v_{z}\right)$ is the three-dimensional molecular velocity, and $h^{\prime}$ is the perturbed distribution function describing the derivation of the system state from the global Maxwellian state $f_{e q}$ :

$$
f_{e q}(\mathbf{v})=\pi^{-3 / 2} \exp \left(-v^{2}\right)
$$

Note that to linearize the Boltzmann equation and various kinetic model equations (such as the Shakhov equation [22]), usually, the distribution function is expressed as $f=f_{e q}\left(1+h^{\prime}\right)$, which makes the LBE elegant and calculation simple. Unfortunately, this kind of linearization does not allow the LBE to be solved numerically by the fast spectral method [23]; actually, the fast spectral method only works if we express the distribution function in the form of Eq. (6). So the unusual linearization given by Eq. (6) is used. The perturbed distribution function $h^{\prime}$ satisfying $\left|h^{\prime} / f_{e q}\right| \ll 1$ is governed by the following LBE:

$$
\frac{\partial h^{\prime}}{\partial t}+v_{x} \frac{\partial h^{\prime}}{\partial x}+v_{y} \frac{\partial h^{\prime}}{\partial y}=\iint B\left(\theta,\left|\mathbf{v}-\mathbf{v}_{*}\right|\right)\left[f_{e q}\left(\mathbf{v}^{\prime}\right) h^{\prime}\left(\mathbf{v}_{*}^{\prime}\right)+f_{e q}\left(\mathbf{v}_{*}^{\prime}\right) h^{\prime}\left(\mathbf{v}^{\prime}\right)-f_{e q}(\mathbf{v}) h^{\prime}\left(\mathbf{v}_{*}\right)-f_{e q}\left(\mathbf{v}_{*}\right) h^{\prime}(\mathbf{v})\right] d \Omega d \mathbf{v}_{*} .
$$

Here, the left-hand-side of Eq. (8) describes the free streaming of gas molecules, while the right-hand-side of Eq. (8) is the linearized Boltzmann collision operator; $\mathbf{v}$ and $\mathbf{v}_{*}$ are the pre-collision velocities of the first and second gas molecules, respectively, while $\mathbf{v}^{\prime}$ and $\mathbf{v}_{*}^{\prime}$ are the corresponding post-collision velocities; they are related to each other as:

$$
\begin{aligned}
\mathbf{v}^{\prime} & =\frac{\mathbf{v}+\mathbf{v}_{*}}{2}+\frac{\left|\mathbf{v}-\mathbf{v}_{*}\right|}{2} \Omega \\
\mathbf{v}_{*}^{\prime} & =\frac{\mathbf{v}+\mathbf{v}_{*}}{2}-\frac{\left|\mathbf{v}-\mathbf{v}_{*}\right|}{2} \Omega
\end{aligned}
$$

where $\Omega$ is the unit vector along the direction of the postrelative velocity $\mathbf{v}^{\prime}-\mathbf{v}_{*}^{\prime} ; \theta$ is the deflection angle that satisfies $\cos \theta=\Omega \cdot\left(\mathbf{v}-\mathbf{v}_{*}\right) /\left|\mathbf{v}-\mathbf{v}_{*}\right|$. Finally, $B(\theta, \mid \mathbf{v}-$ $\left.\mathbf{v}_{*} \mid\right)$ is the collision kernel, which is determined by the intermolecular potential. Detailed forms of $B\left(\theta,\left|\mathbf{v}-\mathbf{v}_{*}\right|\right)$ is complicated for Lennard-Jones potentials [24, 25], so here we use the following special form $[23,26]$ :

$$
B\left(\theta,\left|\mathbf{v}-\mathbf{v}_{*}\right|\right)=C_{\alpha}\left|\mathbf{v}-\mathbf{v}_{*}\right|^{\alpha} \sin ^{\frac{\alpha-1}{2}}(\theta),
$$

where $C_{\alpha}$ and $\alpha$ are two constants, and the shear viscosity of the gas is proportional to the temperature raised to the 
power of $1-\alpha / 2$ (the same relation to that of power-law potentials). Specifically, the hard-sphere and Maxwellian gas molecules have $\alpha=1$ and 0 , respectively. Numerical results below in Sec. III will show that the detailed form of $B\left(\theta,\left|\mathbf{v}-\mathbf{v}_{*}\right|\right)$ has very limited influence on macroscopic flow quantities such as the gas pressure.

We are interested in the state when the harmonic oscillation in the gas has been fully established, so that the state of the gas varies with the same frequency as the oscillating plate. In this case, the perturbed distribution function $h^{\prime}$ can be expressed as

$$
h^{\prime}=\Re[\exp (i \omega t) h(\mathbf{v}, x, y)] \frac{U_{0}}{v_{m}},
$$

where $h$ is the complex perturbation function, governed by the following time-independent $\operatorname{LBE}[21,23]$ :

$$
i S h+v_{x} \frac{\partial h}{\partial x}+v_{y} \frac{\partial h}{\partial y}=\mathcal{L}\left(f_{e q}, h\right)-\nu(\mathbf{v}) h,
$$

where the equilibrium collision frequency is

$$
\nu(\mathbf{v})=\iint B\left(\theta,\left|\mathbf{v}-\mathbf{v}_{*}\right|\right) f_{e q}\left(\mathbf{v}_{*}\right) d \Omega d \mathbf{v}_{*},
$$

and the gain part of the linearized Boltzmann collision operator is

$$
\begin{aligned}
\mathcal{L}\left(f_{\text {eq }}, h\right)=\iint & d \Omega d \mathbf{v}_{*} B\left(\theta,\left|\mathbf{v}-\mathbf{v}_{*}\right|\right) \\
& \times\left[2 f_{\text {eq }}\left(\mathbf{v}^{\prime}\right) h\left(\mathbf{v}_{*}^{\prime}\right)-f_{e q}(\mathbf{v}) h\left(\mathbf{v}_{*}\right)\right] .
\end{aligned}
$$

Note that in writing Eqs. (12)-(14), the molecular velocity $\mathbf{v}$ and spatial variables $x$ and $y$ have been normalized by $v_{m}$ and $L$, respectively. And the constant $C_{\alpha}$ in Eq. (10) is now related to the Knudsen number as $[23,26]$

$$
C_{\alpha}=\frac{5}{2^{\alpha+11 / 2} \Gamma^{2}\left(\frac{\alpha+7}{4}\right) K n},
$$

where $\Gamma$ is the gamma function.

The problem is symmetric about the horizontal line $y=A / 2$, i.e. $h\left(x, y, v_{x}, v_{y}, v_{z}\right)=h\left(x, A-y, v_{x},-v_{y}, v_{z}\right)$. Therefore, only the bottom half domain $(0 \leq x \leq 1$, $0 \leq y \leq A / 2)$ is simulated, with the following diffuse boundary conditions at the left, bottom, and right walls:

$$
\frac{h}{f_{e q}}=\left\{\begin{array}{l}
\sqrt{\pi}+2 v_{x}-2 \sqrt{\pi} \int_{v_{x}<0} v_{x} h d \mathbf{v} ; x=0, v_{x}>0 \\
-2 \sqrt{\pi} \int_{v_{y}<0} v_{y} h d \mathbf{v} ; y=0, v_{y}>0, \\
2 \sqrt{\pi} \int_{v_{x}>0} v_{x} h d \mathbf{v} ; x=1, v_{x}<0 .
\end{array}\right.
$$

When $h$ is obtained, the perturbed pressure field (normalized by $2 n_{0} k_{B} T_{0} U_{0} / v_{m}$ ) in the $x$ direction can be calculated by $P_{x x}(t, x, y)=\Re\left[\exp (i \omega t) p_{x x}(x, y)\right]$, where $p_{x x}(x, y)=\int v_{x}^{2} h(\mathbf{v}, x, y) d \mathbf{v}$. We are most interested in the average gas pressure defined below:

$$
\bar{p}(x)=\frac{\int_{0}^{A} p_{x x}(x, y) d y}{A} .
$$

The amplitude of the average pressure is defined as $|\bar{p}(x)|$, while the phase of the average pressure is calculated by the four-quadrant inverse tangent $\phi=\operatorname{atan} 2(\Im(\bar{p}), \Re(\bar{p}))$, where $\Im$ represents the imaginary part of a complex number. The phase unwrapping algorithm in Matlab is used to calculate the phase of the average pressure in the spatial domain $0 \leq x \leq 1$.

\section{ANALYTICAL AND NUMERICAL METHODS}

The analytical solution for the average pressure can be obtained when the oscillation frequency of the plate is much larger than the mean molecular collision frequency (which is at the order of $n_{0} k_{B} T_{0} / \mu$ ), i.e. when $K n S \gg 1$. In this case, the term in the right-hand-side of Eq. (12) can be neglected. Integrating Eq. (12) with respect to $y$ and introducing a new distribution function

$$
g(x, \mathbf{v})=\frac{\int_{0}^{A} h(x, y, \mathbf{v}) d y}{A},
$$

we obtain

$$
i S g+v_{x} \frac{\partial g}{\partial x}=v_{y} \frac{h(y=0)-h(y=A)}{A} .
$$

We notice that the last term in Eq. (19) can also be neglected when $S \gg 1$. Therefore, we obtain

$$
i S g+v_{x} \frac{d g}{d x}=0 .
$$

From boundary conditions (16) and the definition of $g$ in Eq. (18), the boundary condition for $g$ at $x=0$ and $v_{x}>0$ is

$$
g(0, \mathbf{v})=\left(\sqrt{\pi}+2 v_{x}-2 \sqrt{\pi} \int_{v_{x}<0} v_{x} g d \mathbf{v}\right) f_{e q}(\mathbf{v})
$$

while that at $x=1$ and $v_{x}<0$ is

$$
g(1, \mathbf{v})=2 \sqrt{\pi} f_{e q}(\mathbf{v}) \int_{v_{x}>0} v_{x} g d \mathbf{v}
$$

Hence the analytical solution for $g$ reads

$$
g(x, \mathbf{v})= \begin{cases}\left(2 v_{x}+\nu_{L}\right) f_{e q}(\mathbf{v}) \exp \left(-i \frac{S x}{v_{x}}\right) ; & v_{x}>0 \\ \nu_{R} f_{e q}(\mathbf{v}) \exp \left[-i \frac{S(x-1)}{v_{x}}\right] ; & v_{x}<0\end{cases}
$$


where

$$
\begin{aligned}
\nu_{L} & =\frac{\sqrt{\pi}+8 I_{1}(i S) I_{2}(i S)}{1-4 I_{1}(i S)}, \\
\nu_{R} & =\frac{2 \sqrt{\pi} I_{1}(i S)+4 I_{2}(i S)}{1-4 I_{1}(i S)},
\end{aligned}
$$

with $I_{m}(z)=\int_{0}^{\infty} c^{m} \exp \left(-c^{2}-z / c\right) d c$.

The average pressure $\bar{p}(x)=\int v_{x}^{2} g(x, \mathbf{v}) d \mathbf{v}$ is then calculated to be

$$
\bar{p}(x)=\frac{\nu_{R}}{\sqrt{\pi}} I_{2}[i S(x-1)]+\frac{2}{\sqrt{\pi}} I_{3}(i S x)+\frac{\nu_{L}}{\sqrt{\pi}} I_{2}(i S x) .
$$

Note that the above analytical solution is exactly the same as that for the limiting case of $A=\infty$, i.e. onedimensional sound wave propagating between two infinite parallel plates [12]. It shows when $K n S \gg 1$ and $S \gg 1$, the average pressure $\bar{p}$ has nothing to do with the cavity aspect ratio $A$.

When $S \rightarrow \infty$, the average pressure at $x=0$ (related to the damping force exerting on the oscillating plate) is

$$
\bar{p}(0) \rightarrow \frac{1}{\sqrt{\pi}}+\frac{\sqrt{\pi}}{4},
$$

while the average pressure exerting on the right plate is $\bar{p}(1) \rightarrow 0$.

When the oscillation frequency is not high enough, the LBE must be solved numerically. We adopt the following iterative scheme to solve Eq. (12):

$$
(i S+\nu) h^{n+1}+v_{x} \frac{\partial h^{n+1}}{\partial x}+v_{y} \frac{\partial h^{n+1}}{\partial y}=\mathcal{L}\left(f_{e q}, h^{n}\right),
$$

where the superscript $n$ denotes the iteration step; spatial derivatives $\partial h / \partial x$ and $\partial h / \partial y$ are approximated by the second-order upwind finite difference; $\nu(\mathbf{v})$ and $\mathcal{L}\left(f_{\text {eq }}, h\right)$ defined in Eqs. (13) and (14) are approximated by the fast spectral method [23]. The iteration is terminated when the relative error between two consecutive iteration steps, $\int\left|V^{n+1}-V^{n}\right|^{2} d x d y / \int\left|V^{n}\right|^{2} d x d y$ (where $V$ is the macroscopic quantity such as the density, velocity, and pressure), is less than $10^{-10}$.

In numerical simulations, the three-dimensional molecular velocity space $\mathbf{v}$ is represented by discrete velocities: the velocity component $v_{z}$ is represented by 24 uniform discrete points in the region of $[-6,6]$, while the velocity components $v_{x}$ and $v_{y}$ are represented by $N_{v}$ non-uniform points in each direction:

$$
v_{x, y}=\frac{4}{\left(N_{v}-1\right)^{3}}\left(-N_{v}+1,-N_{v}+3, \cdots, N_{v}-1\right)^{3},
$$

where most of the discrete velocities are located near $v_{x, y}=0$, to capture the large discontinuities and rapid variations, if any, in the distribution function. We choose $N_{v}=48$ (or 96) when $K n=1$ and 0.1 (or $K n=10$ ).
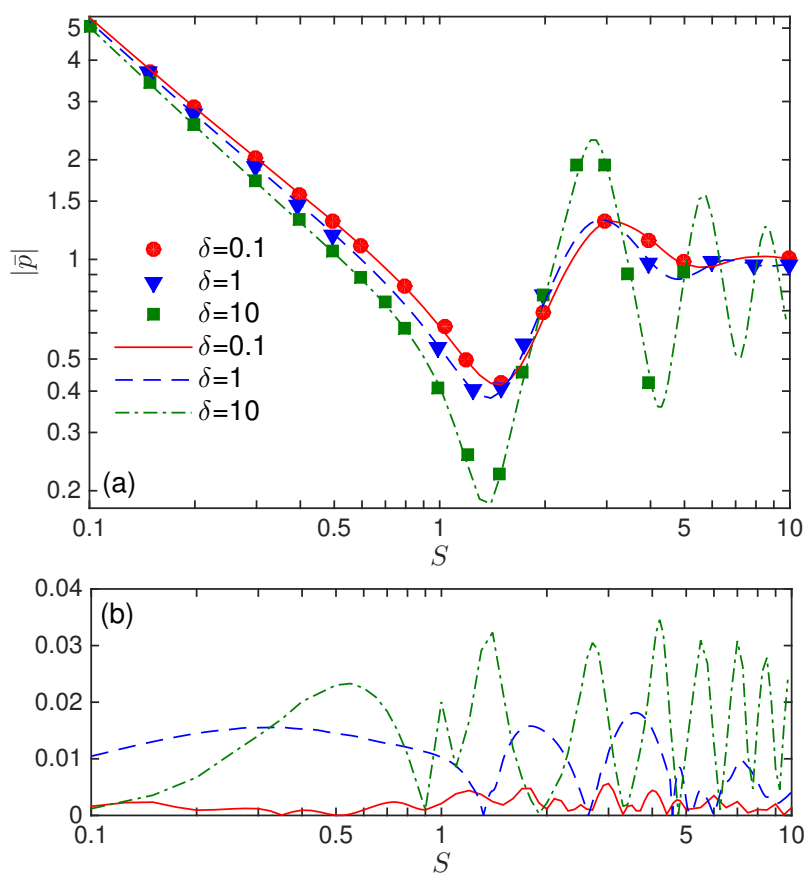

FIG. 2. (Color online) (a) The amplitude of the average pressure at the oscillating plate when $A=\infty$ : comparison between the results of the LBE for hard-sphere molecules (lines) and the Shakhov kinetic model (symbols, adopted from Fig. 1 in Ref. [12]). Note that here $\delta=\sqrt{\pi} / 2 K n S$ and $S$ are equivalent to the $\theta$ and $L$ defined in Ref. [12], respectively. (b) The absolute value of the relative difference in $|\bar{p}(0)|$ between the LBE for the hard-sphere and Maxwellian molecules.

Similarly, the physical space $x$ and $y$ is divided into $N_{x} \times N_{y}$ nonuniform cells, with most of the points located near the cavity walls [23]:

$$
\begin{aligned}
& x=\left(10-15 s_{x}+6 s_{x}^{2}\right) s_{x}^{3}, \quad s_{x}=\frac{\left(0,1, \cdots, N_{x}\right)}{N_{x}}, \\
& y=\left(10-15 s_{y}+6 s_{y}^{2}\right) s_{y}^{3} A, s_{y}=\frac{\left(0,1, \cdots, N_{y}\right)}{2 N_{y}} .
\end{aligned}
$$

We choose $N_{x}=50$, and $N_{y}=50$ when the cavity aspect ratio $A \leq 1$ and $N_{y}=70$ when $A=2$. The numerical method has been proven accurate $[21,23]$, in a manner that doubling the number of discretization points in both physical and velocity spaces produces relative differences in macroscopic quantities (such as the amplitude of the average pressure) less than $0.5 \%$.

To demonstrate the accuracy of our numerical method, we consider the sound propagation between two parallel plates (i.e. $A=\infty$ ), and compare the numerical results of the LBE for hard-sphere molecules with those [12] of the Shakhov kinetic model equation [22], in Fig. 2(a). In the numerical simulation, the term $v_{y} \partial h / \partial y$ in Eq. (12) is dropped. When $\delta=\sqrt{\pi} / 2 K n S=0.1$ and 1 , the physical space $0 \leq x \leq 1$ is divided into 50 nonuniform cells according to Eq. (29), while $v_{x}$ is represented by 96 nonuniform points according to Eq. (28), because the Knudsen 

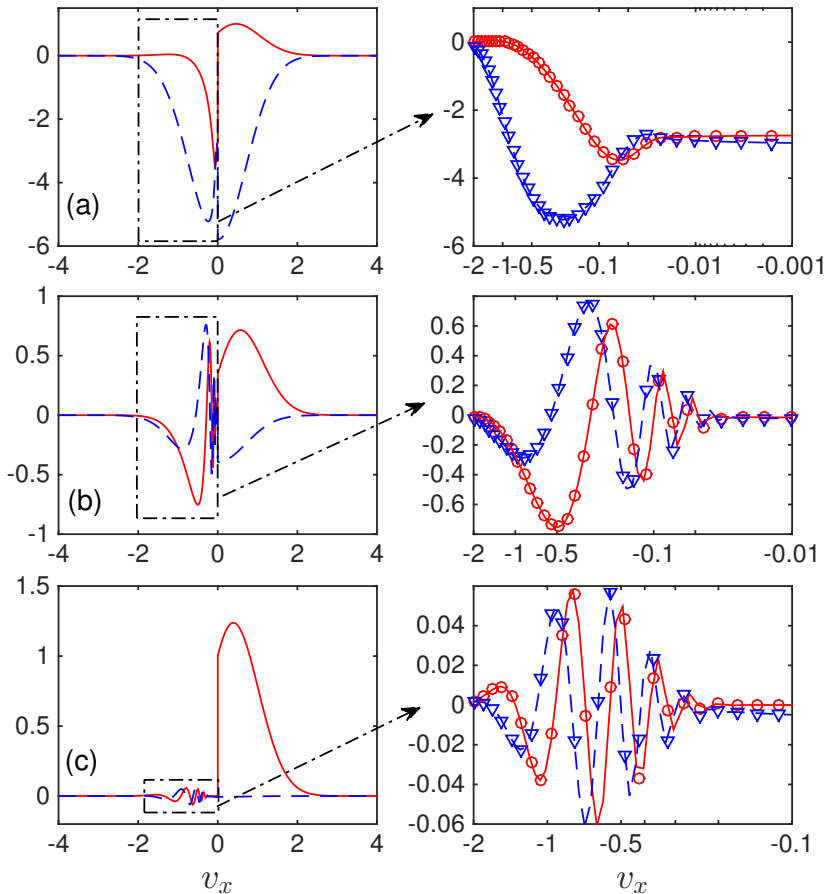

FIG. 3. (Color online) The marginal perturbed distribution function at the oscillating plate, $\iint h(x=0, \mathbf{v}) d v_{y} d v_{z}$, when $A=\infty, K n=5 \sqrt{\pi}$, and the Strouhal number is (a) $S=0.1$, (b) $S=1$, and (c) $S=10$. Large discontinuities and rapid oscillations near $v_{x}=0$ can be clearly seen. Solid lines (or circles) and dashed lines (or triangles) show the real and imaginary parts of the distribution function, respectively, when $N_{v}=192$ (or 96). Note that following the rescaling of the perturbed distribution function in Eq. (11), $\left|h / f_{e q}\right|$ is not necessary far less than unitary.

numbers are large. When $\delta=10$, the physical space is divided into 150 nonuniform cells, and $v_{x}$ is discretized by 48 nonuniform points. The comparison in Fig. 2(a) shows that our method has good accuracy.

We have also solved the LBE for Maxwellian molecules, and found that the difference to that of the hard-spheres molecules is very small. For instance, the relative difference in the amplitude of the average gas pressure exerted on the oscillating plate, over a wide range of $S$ and $K n S$, is within $4 \%$, see Fig. 2(b). This means that the influence of the intermolecular potential is negligible; hence in the following paper, the LBE for the hard-sphere molecules is used.

It is worthy mentioning that at large values of $K n$ and $S$, perturbed distribution functions near cavity walls not only have large discontinuities at $v_{x}=0$, but also oscillate rapidly as $v_{x}$ changes, see the marginal distribution function in Fig. 3, where the distribution function becomes more and more complicated when $S$ increases. This poses a great challenge to the numerical simulation. To reduce the computational cost, Kalempa and Sharipov first introduced additional distribution functions to cast the three-dimensional molecular velocity space to onedimensional, and then they split the solution into two parts: the most oscillatory part of the solution is obtained analytically [12, 27], while the less oscillatory part is solved numerically, by using a large number of uniformly discretized velocities. This method, however, may not work in the two-dimensional cavity flow due to the huge computational cost. Alternatively, on noticing that large discontinuities and rapid variations in distribution functions occur near $v_{x}=0$ for the one-dimensional flow and $v_{x}, v_{y}=0$ for the two-dimensional flow, we adopt the nonuniform discrete velocities, given by Eq. (28), to tackle this problem. The numerical example in Fig. 3 shows that 96 nonuniform discrete velocities in the $v_{x}$ direction can well capture the oscillatory behavior in the distribution function; as a matter of fact, macroscopic flow quantities such as the average gas pressure do not change (up to the fourth decimal) when increasing the number of discrete velocities from $N_{v}=96$ to 192 .

\section{NUMERICAL RESULTS}

Now we employ the numerical simulation to investigate the behavior of the average gas pressure and sound speed in the free-molecular, transition, and slip flow regimes, over the whole range of the Strouhal number and a wide range of the cavity aspect ratio.

\section{A. The average gas pressure}

We first consider the free-molecular flow with $K n$ being infinity, where Eq. (12) is solved by setting the righthand-side term to zero. Profiles of the average pressure (both amplitude and phase) at three typical Strouhal numbers $S$ are shown in Fig. 4. We choose $S=1.5$, 3 , and 9 , because they are respectively close to the first anti-resonance and resonance frequencies, and the high oscillation frequency limit, see Fig. 5. It is clear that the cavity aspect ratio $A$ has a strong influence on the average gas pressure. When $A$ is small, say $A=0.5,0.25$, or 0.125 , the amplitude of the average pressure decreases as the distance to the sound source $x$ increases (except that it increases slightly near the right plate). This implies that most of the sound energy concentrates near the sound source: the higher the oscillation frequency and the smaller the aspect ratio, the more energy concentrates. However, when $A$ is large, say $A=2$ and $\infty$, only at large oscillation frequencies does the amplitude of the average pressure decrease with $x$ in general; while at $S=1.5$ the amplitude increases as the distance to the sound source $x$ decreases, and at $S=3$ the amplitude first decreases and then increases as $x$ increases. From Fig. 4(c) we see that at $S=9$ the average pressure at the oscillating plate is already very close to the asymptotic value in Eq. (26), however, the average pressure exerting on the right plate is still far away from the asymptotic 

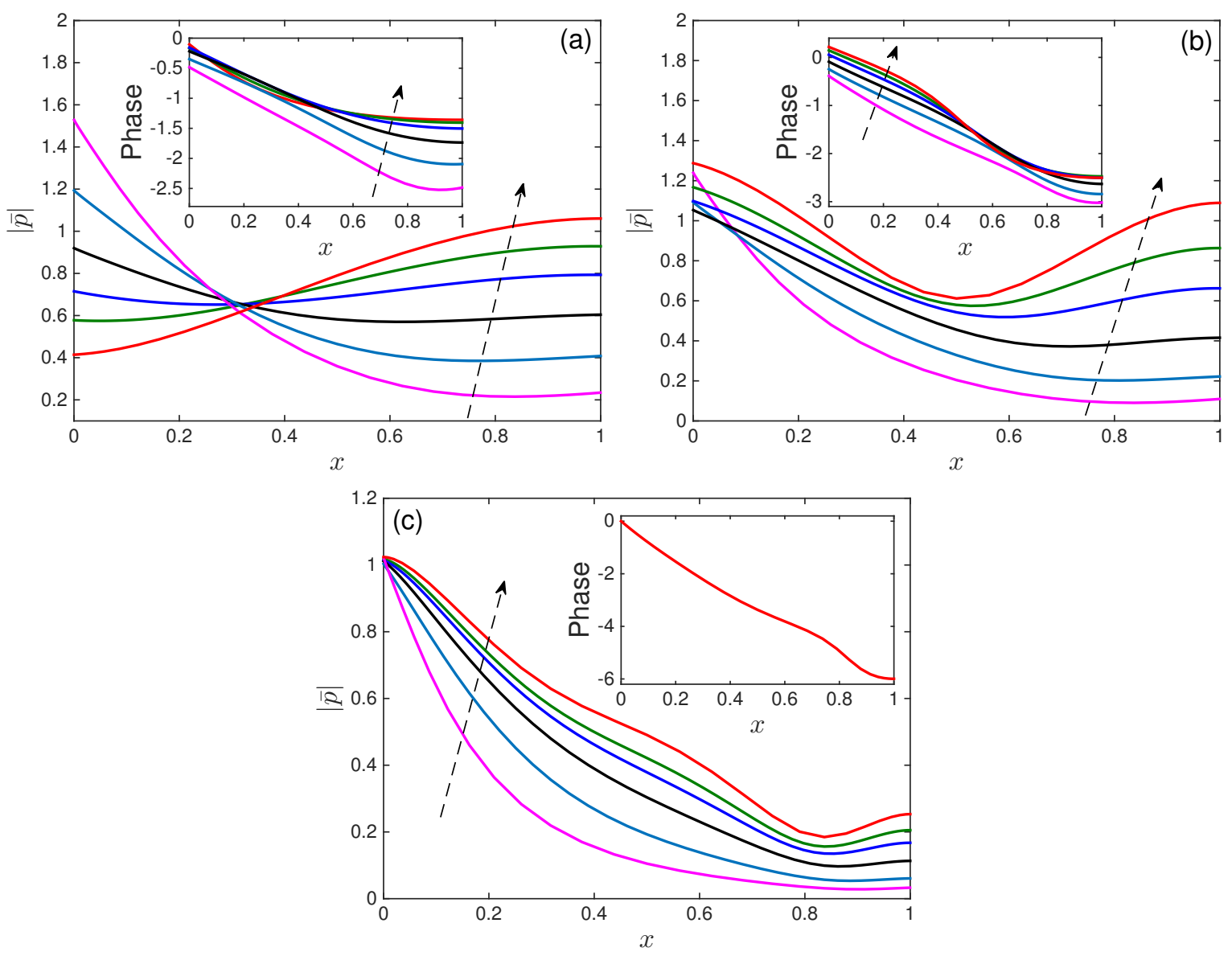

FIG. 4. (Color online) The amplitude and phase of the average pressure in the free-molecular flow regime with $1 / K n=0$, when the Strouhal number is (a) $S=1.5$, (b) $S=3$, and (c) $S=9$. Along the direction of the arrow, the cavity aspect ratios are $0.125,0.25,0.5,1,2$, and infinity, respectively. In the inset of (c), the phases of the average pressure for different aspect ratios are nearly indistinguishable, so only the phase for $A=\infty$ is shown.

value zero, indicating that the high oscillation frequency limit has not been fully reached. From the three insets in Fig. 4 we can see that the phase of the average pressure is not a linear function of $x$ in the whole domain, which means that the phase speed of the sound wave varies by location.

Figure 5 depicts the average pressure on the oscillating plates, as a function of the Strouhal number $S$, at six different cavity aspect ratios. At large values of $A$, as the Strouhal number increases, the amplitude of the average pressure first decreases, then oscillates several times, and finally approaches to a constant value given by Eq. (26) when the oscillation frequency is high. As the aspect ratio decreases, the oscillatory behavior of the average pressure, as a function of $S$, becomes weaker and weaker. When the aspect ratio is small enough, say $A=0.25$ and 0.125 , the oscillatory behavior is completely eliminated. In this case, the increase of the Strouhal number leads to the decrease of the amplitude of the average pressure; and the smaller the value of aspect ratio $A$, the slower the decrease. The behavior of the phase of the average pressure as a function of the Strouhal number is, however, in the reverse direction to the amplitude of the average pressure. That is, while the amplitude decreases (or increases) with increasing $S$, the phase increases (or decreases), which approaches zero when the oscillation frequency is high.

In the one-dimensional geometry (corresponding to $A$ being infinity), the oscillatory behavior of the average pressure as the function of $S$ can be explained by the sound interference $[18,21]$. In the free-molecular flow regime, the binary collision is negligible. Using the method of characteristics, Eq. (12) is rewritten as

$$
i S h+\xi \frac{\partial h}{\partial s}=0, \quad \xi=\sqrt{v_{x}^{2}+v_{y}^{2}},
$$

where $\xi$ is the molecular speed and $s$ is the coordinate along the characteristic line [28]. Choosing $\xi$ to be the most probable speed, Eq. (30) becomes $i S h+\partial h / \partial s=0$, which shows that the phase of the distribution function remains unchanged after gas molecules have traveled a distance of $2 \pi / S$. Let us consider molecules leaving the 

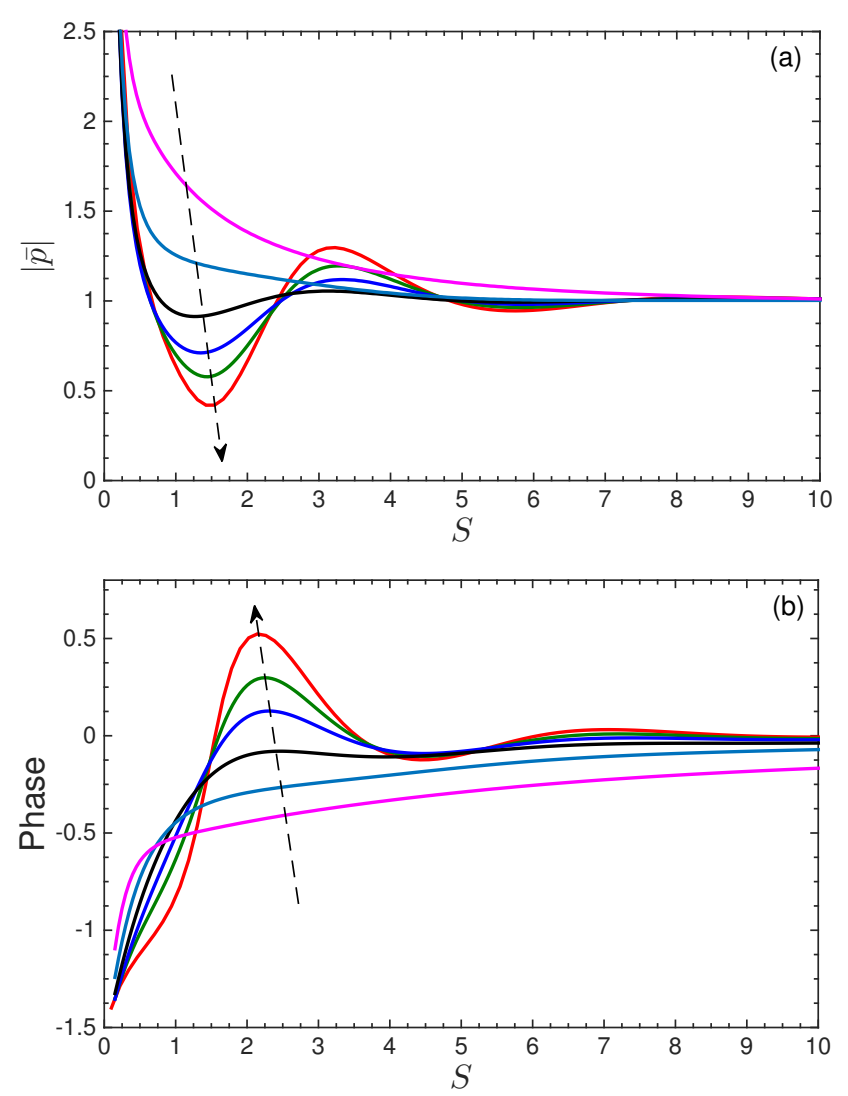

FIG. 5. (Color online) The amplitude (a) and phase (b) of the average pressure at the oscillating plate in the free-molecular flow regime with $1 / K n=0$. Along the direction of the arrow, the cavity aspect ratios are $0.125,0.25,0.5,1,2$, and infinity, respectively.

left plate at $x=0$, hitting the right plate at $x=1$, then being reflected and finally returning to the point from which they left, see type I interference in Fig. 1. The distance gas molecules have traveled is about 2. Therefore, at the resonance frequency

$$
S_{r, 1} \simeq m_{1} \pi, \quad m_{1}=0,1,2, \cdots,
$$

the two distribution functions corresponding to molecules moving leftwards and rightwards have the same phase, so that the gas pressure exerting on the oscillating plate is maximum; similarly, at the anti-resonance frequency

$$
S_{a, 1} \simeq \frac{\left(2 n_{1}+1\right)}{2} \pi, \quad n_{1}=0,1,2, \cdots,
$$

the gas pressure on the oscillating plate is minimum.

The above two equations can roughly explain the first anti-resonance $(S=1.5 \simeq \pi / 2)$ and resonance $(S=$ $3.2 \simeq \pi$ ) frequencies in Fig. 5 , when the cavity aspect ratio $A$ is infinity. It can be even applied to the cases of $A=2$ and 1 .

However, Eqs. (31) and (32) derived from the type I interference has noting to do with the cavity aspect ratio and can not explain the behavior of average pressure
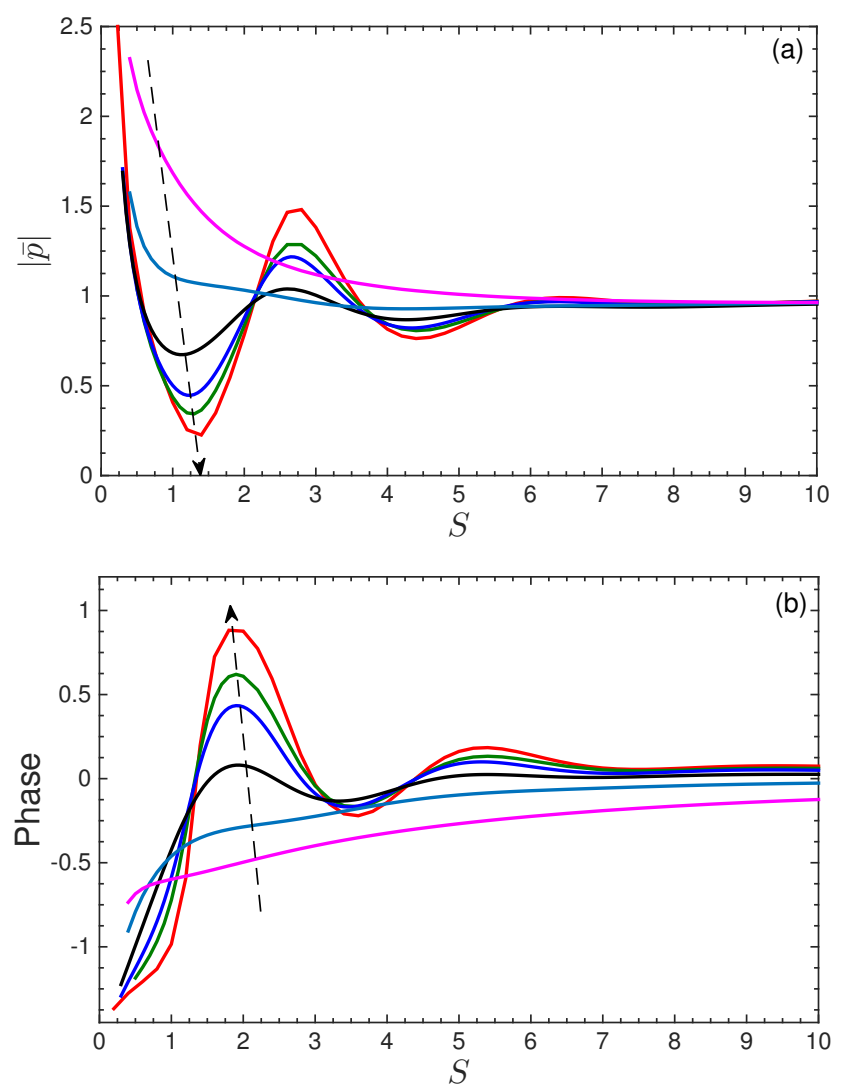

FIG. 6. (Color online) The amplitude (a) and phase (b) of the average pressure at the oscillating plate when $K n=0.1$. Along the direction of the arrow, the cavity aspect ratios are $0.125,0.25,0.5,1,2$, and infinity, respectively.

when $A$ is small, say $A=0.25$ and 0.125 . This is because when $A$ is small, gas molecules reflected by the left plate has very small chances of hitting the right plate but are more likely to hit the top and bottom plates, a scenario where the type II interference becomes dominant, see Fig. 1. Consider molecules leaving the left plate with velocities nearly parallel to the left plate, hitting the top (or bottom) plates, then being reflected and hitting the bottom (or top) plate, and finally returning to the point from which they left. The distance they have traveled is about $2 A$. Therefore, the pressure on the oscillating plate is maximum (minimum) at the resonance (anti-resonance) frequency:

$$
\begin{aligned}
& S_{r, 2} \simeq \frac{m_{2}}{A} \pi, \\
& S_{a, 2} \simeq \frac{\left(2 n_{2}+1\right)}{2 A} \pi,
\end{aligned}
$$

where $m_{2}$ and $n_{2}$ are non-negative constants.

The type II anti-resonance can be used to qualitatively explain the monotonically decreasing of the amplitude of the average pressure when $S$ increases. For example, when $A=0.25$, the first type II anti-resonance frequency 
is around $2 \pi$, which means that the amplitude of the average pressure decreases with $S$ when $S \lesssim 2 \pi$. And since $S=2 \pi$ is very close to the high oscillation frequency limit, the amplitude of the average pressure continues to decrease to the asymptotic limit given by Eq. (26) when $S$ further increases. Type II anti-resonance can also be used to explain why the amplitude of the average pressure decrease more slowly at smaller cavity aspect ratios: the smaller the aspect ratio $A$, the larger the first anti-resonance frequency $S_{a, 2}$, and therefore the slower the decaying of the amplitude of the average pressure with respect to $S$.

We then investigate the average pressure on the oscillating plate in the transition and slip flow regimes. To this end, we choose two representative Knudsen numbers, $K n=1$ and 0.1 . The results of $K n=0.1$ are presented in Fig. 6, while that of $K n=1$ are not shown as they are very close to those in Fig. 5 in the free-molecular flow regime. Comparisons between Figs. 5 and 6 illustrate that, as $K n$ decreases, the amplitude of the average pressure oscillates more strongly with $S$ when $A$ is large. This is comprehensible because smaller $K n$ leads to smaller dissipation and hence larger oscillation amplitude; in fact, the oscillation is so large than the second anti-resonance can be easily observed in Fig. 6: interestingly, we found that the second anti-resonance frequency can be predicted perfectly by $S_{a, 1}$ in Eq. (32) with $n_{1}=1$, when the aspect ratio $A$ is large. We also found that the first anti-resonance and resonance frequency decreases slightly with $K n$.

\section{B. The sound speed near the source}

We now investigate how the cavity aspect ratio affect the sound speed. Although from the insets in Fig. 4 we see that the phases of the average pressure are not linear functions of $x$ in the whole domain, they vary almost linearly with $x$ close to the sound source, which implies a constant sound speed. Hence we calculate the phase speed of the sound near the sound source as

$$
v_{p h}=-\frac{S}{\left.(\partial \phi / \partial x)\right|_{x=0}},
$$

where $\phi$ is the phase of the average gas pressure $\bar{p}$. Note that the sound speed has been normalized by the most probable speed $v_{m}$.

It will be interesting to note that, for rarefied gas flows, the differential phase speed defined by Eq. (34) could be negative at some location; for instance, see the variation of the phase near $x=1$, at small values of the cavity aspect ratio, in the inset of Fig. 4(a). In this case, the integral phase speed, defined by Eqs. (19) and (21) in Ref. [12], is introduced to calculate the sound speed at the receptor. In the present paper, however, we find that the differential phase speed is always positive near the sound source (see the insets in Fig. 4 near $x=0$ ), so only Eq. (34) is considered.
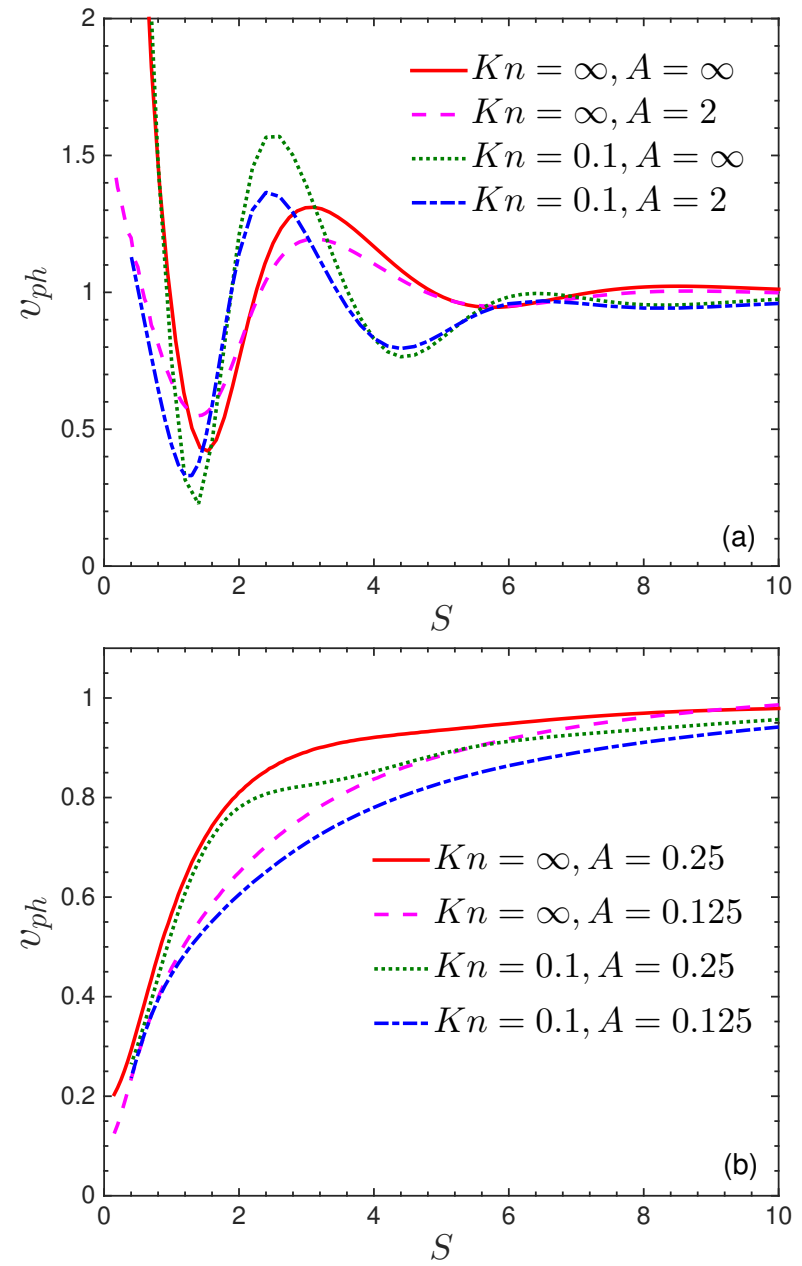

FIG. 7. (Color online) The variation of the sound phase speed near the sound source with the Strouhal number, at large (a) and small (b) values of the cavity aspect ratio.

The analytical solution for the phase speed can also be obtained when $K n S \gg 1$ and $S \gg 1$. From Eq. (25) we find that the average pressure near the sound source (when $S x \rightarrow 0$ ) is

$$
\bar{p}(x)=\frac{1}{\sqrt{\pi}}+\frac{\sqrt{\pi}}{4}-i S x \simeq 1-i S x .
$$

Thus, the phase of the average pressure near the oscillating plate is $-S x$, and the phase speed near the sound source equals the most probable speed.

Typical profiles of the sound speed are depicted in Fig. 7 when $K n=\infty$ and 0.1 . It is clearly seen that, when $S$ is large, the phase speed approaches the most probable speed. From Fig. 7(a) we see that, when the cavity aspect ratio $A$ is large, the sound speed exhibits the same oscillatory behavior (in-phase) as the amplitude of the average pressure in Figs. 5 and 6 . From this it may be concluded that the more (less) sound energy concentrated near the oscillating plate, the larger (smaller) the sound speed. However, when $A$ is small, the sound speed 
increases monotonically with $S$ [Fig. 7(b)], whereas the amplitude of the average pressure decreases monotonically; that is, the phase speed and the amplitude of the average pressure near the sound source are out-of-phase. We also find that, when the values of $K n$ and $S$ are fixed, smaller value of the cavity aspect ratio leads to smaller value of the sound speed, which is consistent with the discovery in narrow channels [13].

\section{CONCLUSIONS}

The linearized Boltzmann equation has been solved analytical and numerically to study the sound propagation inside rectangular cavities, where one of the cavity walls oscillates and acts as the sound source. It has been found that the damping force (average gas pressure) exerting on the oscillating plate and the sound speed are significantly affected by the aspect ratio of the cavity. When the aspect ratio is larger than the threshold value of 0.5 , the damping force and sound speed oscillate in-phase, as the oscillation frequency increases. However, when the aspect ratio is less than 0.5 , the changes of damping force and sound speed are out-of-phase: as the oscillation frequency increases, the damping force decreases, but the sound speed increases. To our knowledge, this exotic behavior of the damping force has not been observed in oscillatory rarefied gas flows before. We attributed this novel phenomenon to a new type of sound interference, occurring in the direction perpendicular to the motion of the oscillating plate. Our proposed simple analytical expressions well predicted the resonance and anti-resonance frequencies.

LW acknowledges the financial support of an ECR International Exchange Award from the Glasgow Research Partnership in Engineering.
[1] G. Karniadakis, A. Beskok, and N. Aluru, Microflows and Nanoflows: Fundamentals and Simulation (Springer, 2005).

[2] M. Gad-el-Hak, "The fluid mechanics of microdevices the Freeman Scholar lecture," J. Fluids Eng. 121, 5-33 (1999).

[3] J. H. Ferziger and H. G. Kaper, Mathematical Theory of Transport Processes in Gases (North-Holland Publishing Company, Amsterdam, 1972).

[4] C. Cercignani, The Boltzmann Equation and its Applications (Springer-Verlag, New York, 1988).

[5] F. Sharipov, Rarefied Gas Dynamics. Fundamentals for Research and Practice (Wiley-VCH, Berlin, 2016).

[6] M. Knudsen, "Die Gesetze der Molekularströmung und der inneren Reibungsströmung der Gase durch Röhren," Ann. Phys. 333, 75-130 (1909).

[7] W. Steckelmacher, "Knudsen flow 75 years on: the current state of the art for flow of rarefied gases in tubes and systems," Rep. Prog. Phys. 49, 1083-1107 (1999).

[8] O. Reynolds, "On certain dimensional properties of matter in the gaseous state," Phil. Trans. R. Soc. Lond. 170, 727-845 (1879).

[9] J. Nabeth, S. Chigullapalli, and A. A. Alexeenko, "Quantifying the Knudsen force on heated microbeams: A compact model and direct comparison with measurements," Phys. Rev. E 83, 066306 (2011).

[10] H. Yamaguchi, M. Rojas-Cárdenas, P. Perrier, I. Graur, and T. Niimi, "Thermal transpiration flow through a single rectangular channel journal," J. Fluid Mech. 744, 169-182 (2014).

[11] F. Sharipov and D. Kalempa, "Numerical modelling of the sound propagation through a rarefied gas in a semiinfinite space on the basis of linearized kinetic equation," J. Acoust. Soc. Am. 124, 1993-2001 (2008).

[12] D. Kalempa and F. Sharipov, "Sound propagation through a rarefied gas confined between source and receptor at arbitrary Knudsen number and sound frequency," Phys. Fluids 21, 103601 (2009).

[13] N. G. Hadjiconstantinou, "Sound wave propagation in transition-regime micro- and nanochannels," Phys. Fluids 14, 802 (2002).

[14] J. H. Park, P. Bahukudumbi, and A. Beskok, "Rarefaction effects on shear driven oscillatory gas flows: A direct simulation Monte Carlo study in the entire Knudsen regime," Phys. Fluids 16, 317 (2004).

[15] D. R. Emerson, X. J. Gu, S. K. Stefanov, Y. H. Sun, and R. W. Barber, "Nonplanar oscillatory shear flow: from the continuum to the free-molecular regime," Phys. Fluids 19, 107105 (2007).

[16] T. Doi, "Numerical analysis of oscillatory Couette flow of a rarefied gas on the basis of the linearized Boltzmann equation," Vacuum 84, 734-737 (2009).

[17] X. J. Gu and D. R. Emerson, "Modeling oscillatory flows in the transition regime using a high-order moment method," Microfluid Nanofluid 10, 389-401 (2011).

[18] L. Desvillettes and S. Lorenzani, "Sound wave resonance in micro-electro-mechanical systems devices vibrating at high frequencies according to the kinetic theory of gases," Phys. Fluids 24, 092001 (2012).

[19] D. Kalempa and F. Sharipov, "Sound propagation through a rarefied gas: Influence of the gas-surface interaction," Int. J. Heat Fluid Flow 30, 190-199 (2012).

[20] Y. W. Yap and J. E. Sader, "Sphere oscillating in a rarefied gas," J. Fluid Mech. 794, 109-153 (2016).

[21] L. Wu, J. M. Reese, and Y. H. Zhang, "Oscillatory rarefied gas flow inside rectangular cavities," J. Fluid Mech. 748, 350-367 (2014).

[22] E. M. Shakhov, "Approximate kinetic equations in rarefied gas theory," Fluid Dynamics 3(1), 112-115 (1968).

[23] L. Wu, J. M. Reese, and Y. H. Zhang, "Solving the Boltzmann equation by the fast spectral method: application to microflows," J. Fluid Mech. 746, 53-84 (2014).

[24] F. Sharipov and G. Bertoldo, "Numerical solution of the linearized Boltzmann equation for an arbitrary intermolecular potential," J. Comput. Phys. 228, 3345-3357 (2009).

[25] A. Venkattraman and A. A. Alexeenko, "Binary scattering model for Lennard-Jones potential: Transport coeffi- 
cients and collision integrals for non-equilibrium gas flow simulations," Phys. Fluids 24, 027101 (2012).

[26] L. Wu, C. White, T. J. Scanlon, J. M. Reese, and Y. H. Zhang, "Deterministic numerical solutions of the Boltzmann equation using the fast spectral method," J. Comput. Phys. (2013).

[27] F. Sharipov and D. Kalempa, "Oscillatory Couette flow at arbitrary oscillation frequency over the whole range of the Knudsen number," Microfluid Nanofluid 4, 363-374 (2008).

[28] S. Varoutis, D. Valougeorgis, and F. Sharipov, "Application of the integro-moment method to steady-state twodimensional rarefied gas flows subject to boundary induced discontinuities," J. Comput. Phys. 227, 6272-6287 (2008). 